\title{
Multi-UAV System Architecture for Environmental Protection Area Monitoring
}

\author{
Vitor G. Santos * Diego S. Pereira ${ }^{* *}$ Pablo J. Alsina * \\ Daniel H. S. Fernandes* Luís B. P. Nascimento* \\ Deyvid L. Leite* Matheus R. Morais* Maurício R. Silva* \\ Elitelma S. Souza ${ }^{* * *}$ \\ * Department of Computer Engineering and Automation, Federal \\ University of Rio Grande do Norte, Natal, RN, (e-mail: \\ vitorgaboardi@ufrn.edu.com, pablo@dca.ufrn.br,lbruno@ufrn.edu.br). \\ ** Applied Computing Studies Group, Federal Institute of Rio Grande \\ do Norte, Parnamirim, RN (e-mail: diego.pereira@ifrn.edu.br) \\ *** Sidia Institute of Science and Technology, Manaus, AM (e-mail: \\ elitelma.s@samsung.com)
}

\begin{abstract}
Environmental Protection Area (APA) are limited regions that have the main goal to conserve natural resources and the existing natural environment. However, controlling illegal anthropogenic actions in APAs is a difficult task, thus a proper monitoring system is required to solve this problem. In this paper we present a new communication architecture made with a multi Unmanned Aerial Vehicles (UAVs) network through XBee modules and an image processing system that uses a pretrained neural network to detect human activities in a Coral Reefs Environmental Protection Area (APARC). Simulations prove that the system is able to change data up to $6.3 \mathrm{~km}$ and the image processing algorithm was able to detect boats satisfactorily.
\end{abstract}

Keywords: Monitoring System, Communication Architecture, UAV, Image Processing, SSD, Environmental Protection Area.

\section{INTRODUCTION}

A nature Conservation Unit (UC) is a restricted area that presents natural features considered to be unique that aims to ensure the protection and conservation of its natural resources (BRASIL, 2000).

Currently, the Brazilian state of Rio Grande do Norte (RN) has $4.5 \%$ of its territory (238 thousand hectares) distributed in eight UCs, where $2.58 \%$ are in the marine ecosystem, $1.08 \%$ in the coastal ecosystem, $0.8 \%$ in the atlantic forest ecosystem and the remaining is in the Caatinga (IDEMA, 2019). The Nucleus of Conservation Unit, which is part of the Institute of Sustainable Development and Environment of Rio Grande do Norte (IDEMA), is responsable for managing the UCs.

Among the UCs established by the state government, we highlight the Coral Reefs Environmental Protection Area (APARC), which has 136 thousand hectares distributed along the coastal strip from the cities of Maxaranguape, Rio do Fogo and Touros. This region is considered to be one of the most biodiverse in the world, therefore, it became an intense tourist center in the RN, where the visitors can perform activities such as underwater diving, artisanal fishing, visit the coral reefs and scientific research. An APARC image is presented in Figure 1.

Unfortunately, tourism can be harmful to the environment and puts pressure on local populations. Therefore, some preservation directives were created based on regulamentations from nature UC which aims to reduce the effects

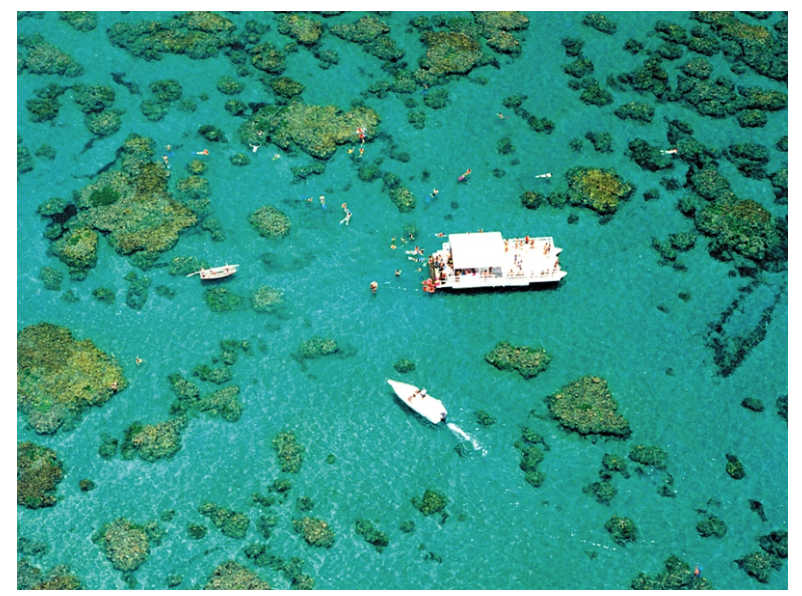

Figure 1. The Coral Reefs Environmental Protection Area (APARC) IDEMA (2019).

of anthropic activities, e.g. restrictions meant to curtail the number of travelers and conduct rules that must be followed within the environment (IDEMA, 2019).

Thus, monitoring actions in real time are important to guarantee the preservation of this UC. Presently, there is a monitoring program operated by APARC, but the extensive area and the limited resources from the government hampers the daily execution.

In this context, we propose a multi-UAV (Unmanned Aerial Vehicle) monitoring system as an alternative to 
detect human activities in forbidden areas. In this paper, we chose to monitor the APARC, a region where the access is not allowed neither by any watercraft nor by people. Therefore, the main goal is to use the UAVs, in scheduled daily rounds, along an embedded image processing system to detect boats in the area and notify a system operator that will put in action a team to investigate occurence.

Our main contribution is the development of a new communication architecture focused in multi-UAV for monitoring that changes data about the detection between the UAVs and a base station (BS). Another contribution is the incorporation of a image processing system in the UAV to detect boats using a SSD neural network.

The following sections are organized as follows: Section 2 presents a brief state of the art about environmental monitoring by UAVs. Section 3 describes the monitoring architecture proposed in this work. Section 4 presents some preliminary results obtained with simulations and, finally, section 5 presents the final considerations.

\section{RELATED WORKS}

Robotic systems are increasingly being used as fundamental tools for data collection, allowing new perspectives and a better understanding of the planet and its environmental processes. It is possible to find robots with diverse capacities, such as: to explore extremely deep parts of the oceans, to track harmful algal proliferations, to monitor climatic variables, to explore volcanoes, among others.

In Dunbabin and Marques (2012), it was discussed significant advances and applications with marine, terrestrial and aerial robotic systems developed for the purpose of environmental monitoring. Hence, a survey of publications was made which categorized in small, medium and large scale systems and the main characteristics of each solution, such as cooperation, remote sensing, interaction between robots and the sensor network, mapping and localization, classification and image processing. From here onwards, it is possible to choose the best strategies according to the environment to be monitored.

The construction and validation of a mobile sensor platform for monitoring the greenhouse effect was done in Roldán et al. (2015). The system consists of a sensory platform, embedded in a small quadrotor and has the main goals of taking measures of temperature, humidity, luminosity and $\mathrm{CO}_{2}$ and plotting maps of these variables.

The visual area coverage problem is treated in Balampanis et al. (2017), which addresses the problems of exact cell decomposition and division of a coastal region for a team of UAVs with an approach that takes into consideration the field of view (FOV) or the sensing radius of the sensors on-board.

Papatheodorou et al. (2017) also address the problem of visual coverage area with an aircraft squadron. However, the authors classify the system components as Mobile Aerial Agents (MAAs). Each MAA is equipped with a face down camera that has a conical field of view which covers all points within a circle on the ground. The diameter of this circle is proportional to the altitude of the MAA, while the quality of the covered area decreases. A distributed control law is developed to maximize a coverage quality criterion by adjusting the spatial coordinates of the MAAs.

In Chen et al. (2014), a survey of several works that use systems composed by UAVs to perform coverage area is presented. The article analyzes the researches in many features, such as the type of coverage adopted, either static or dynamic; existence of connectivity between UAVs; whether the UAV network is homogeneous or heterogeneous; among other items that are important for the study and understanding of the issues presented here.

\section{MULTI-UAV SYSTEM FOR ENVIRONMENTAL PROTECTION AREA MONITORING}

An application for monitoring a region of environmental protection is complex, covers several areas and may have different purposes. This work aims to perform a visual monitoring of a particular area using a fleet of autonomous aircraft to detect boats in forbidden regions.

The proposed multi-UAV system (Figure 2) consists of some aircrafts equipped with camera, GPS, communication interfaces, autopilot and a software embedded in a microcomputer that enables the operation of the system. Each aircraft has a specific coverage area that depends on the scanning strategy adopted. In this work, we consider that the aircrafts are in a formation where they have the same flight speed and operate at the same height.

\subsection{Communication Network}

The proposed multi-UAV system network was physically built based on XBee Pro S3 modules, connected to Raspberry Pi 3 microcomputers with Raspbian Linux operating system. These devices will be embedded in aerial vehicles quadrotor, equipped with GPS and automatic pilot controller board.

There are two ways of communication between the components of the system: in the first one, U2U (UAV to UAV), information is exchanged between the aircrafts that compose the monitoring network; in the second one, U2S (UAV to BS), data is sent from an aircraft to the base station, which is the interface that shows the information captured by the UAVs to the system operator.

The comunication system between the base station and the UAV is made using XBee/PRO-S3 transceivers with power of $250 \mathrm{~mW}$ at the frequency of $920 \mathrm{MHz}$. The system has omnidirectional filamentary antennas with $1.5 \mathrm{dBi}$ gain that provides a satisfatory capacity of data transmission between the U2S, since this configuration allows the base station to receive the same signal power independently of the UAV movement direction. Moreover, the system has a receiver with sensitivity of $-100 \mathrm{dBm}$ that allows an efficient range for the aircrafts to work.

Radio Link: The UAV has a radio link that can send telemetry and images information to the base station. The BS and the aircrafts use the XBee-PRO S3 transceiver which consists of a GFSK modem, a transmitter, a receiver and an omnidirectional antenna. The transmission system works with two settings of baud rate and a minimum sensitivity to maintain the rate, which can be checked in Table 1. 

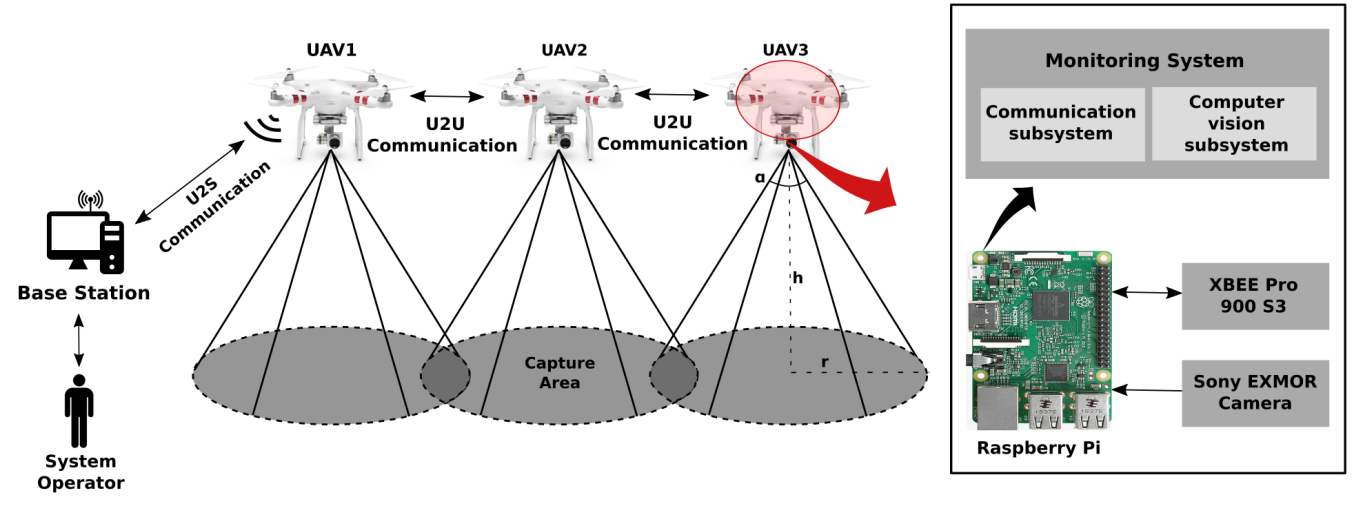

Figure 2. The multi-UAV system architecture for monitoring area.

Table 1. The communication system configuration

\begin{tabular}{|c|c|c|}
\hline $\begin{array}{c}\text { Range } \\
(\mathbf{k m})\end{array}$ & $\begin{array}{c}\text { Transmission rate } \\
(\mathbf{k b} / \mathbf{s})\end{array}$ & $\begin{array}{c}\text { Minimum power } \\
(\mathbf{d B m})\end{array}$ \\
\hline 6.5 & 200 & -101 \\
\hline 15.5 & 10 & -110 \\
\hline
\end{tabular}

Message Structure: The information is transmitted through the DigiMesh network established by the XBee modules and will be performed via API mode. The structured data packets in API mode are called frames. They are sent and received through the serial interface of the device and contain the wireless message itself as well as some extra information such as the destination/source of the data or the signal quality. When a device is in API mode, all data entering and leaving the module through the serial interface is contained in frames that define operations or events within the device. These data is sent through the XBee using hexadecimal characters.

The architecture presented in this paper will be used to send encoded images. Therefore, an image compression and coding algorithm will be performed to convert these images in substrings of up to 256 bytes in size and then send this data over XBee frames.

The images from the monitoring process have a size larger than 256 bytes, therefore several sequential frames have to be used in order to send/receive an image. To organize the incoming data, each frame will have a three-digit identification code (ID). Thus, each frame will have 253 bytes for data and 3 bytes for ID.

\subsection{Image Processing Subsystem}

It is possible to extract information from the images in real time to detect irregularities in the environment using the embedded camera in the UAV, such as the presence of watercraft, people or any specific problem in the region. Hence, it is necessary to develop an algorithm capable of interpreting the content of the image captured by the UAV as faster as possible.

Frame rate: In this work, we need to develop an algorithm that works efficiently and that consumes the less processing time and memory possible, due to the embedded microcontroler limitations. Therefore, we have to chose wisely the period $(\mathrm{T})$ which the embedded camera in the
UAV captures two consecutive images: if a short period of time is chosen, the microcontroler will not be able to process all the information; on the other hand, if a long period of time is chosen, there will be areas that will not be covered by the UAVs squadron.

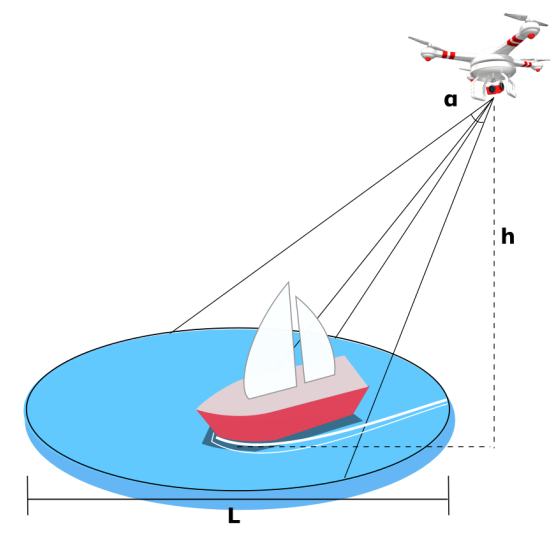

Figure 3. Image Capture Strategy.

The methodology used to capture images is based in Santos et al. (2015) and Silva et al. (2015). The relationship between some variables shown in Figure 3 is provided by equation 1.

$$
L=2 h \tan \left(\frac{\alpha}{2}\right)
$$

where $h$ represents the UAV flight altitude relative to the ground, $\alpha$ is the field of view (FOV) angle of the embedded camera and $L$ is the diameter of the captured area in meters. Moreover, equation 2 relates the time $T$ with $L$ and the UAV velocity $(V)$ :

$$
T \leq \frac{0.8 L}{V}
$$

The lower the value of $T$, the higher the intersection area of two consectuive images obtained by the camera from the same UAV will be, increasing the system reliability since some parts of the image area will be verified more than once.

In the proposed scenario, the UAVs will have $h=90 \mathrm{~m}$ and $\alpha=1.64$ rad which results in a coverage area of 
$L=195 \mathrm{~m}$ for each UAV. In a multi-UAV system with three aircrafts flying side by side the total coverage area will be $L_{\text {total }}=3 L=585 \mathrm{~m}$. However, we will consider a $20 \%$ intersection area between two images, so only $80 \%$ of $\mathrm{L}$ will be covered by the UAV, as shown in equation 2 . As a result, the new $L$ is $156 \mathrm{~m}$ and $L_{\text {total }}=3 L=468 \mathrm{~m}$. The UAVs velocity will be defined as $V=15 \mathrm{~m} / \mathrm{s}$, which results in $T \leq 10.4 \mathrm{~s}$.

Figure 4 illustrates the behavior of the system for image acquisition. $t_{1}, t_{2}$ and $t_{3}$ represent the time that each UAV will capture the first, second and third environment image, respectively. The image $\mathrm{UAV}_{1}$ has $40 \%$ of its area verified twice and image $_{2} \mathrm{UAV}_{1}$ has $60 \%$. Therefore, this strategy will improve the system reliability.

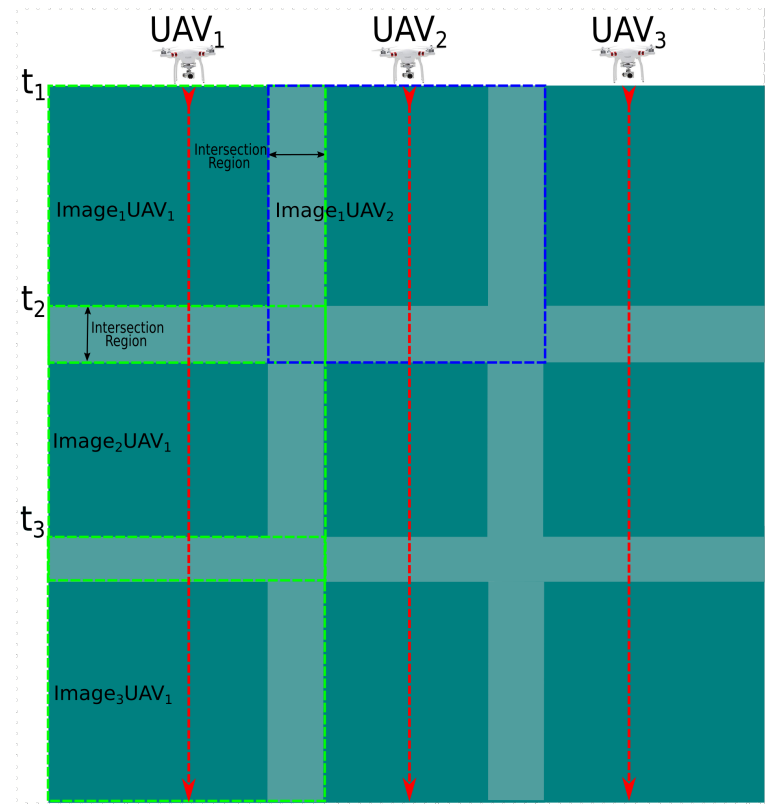

Figure 4. Image Capture Strategy using three UAVs.

Boat Detection: At this point, it is clear the need of an image processing algorithm to detect boats in a forbidden area. In Liu et al. (2016), it is presented a method for detecting objects in images using a single deep neural network. Their approach, called Single Shot Multibox Detector (SSD), discretizes the output space of bounding boxes into a set of default boxes over different aspect ratios and scales per feature map location. SSD is easy to train and straightforward to integrate into systems that require a detection component.

Furthermore, we used the Pascal Visual Object Classes (VOC) [Everingham et al. (2010)], which contains images of many classes, such as people, cars, horses, boats and others, as dataset to train a neural network in order to recognize objects from a number of visual object classes in realistic scenes.

Initially, in our work, we used this pretrained model, i.e., we tried to detect boats using a neural network that was trained to detect several kinds of classes. So, if the model provides an acceptable response in this task, it will corroborate to train our own network using the SSD framework and a dataset of our interest, which will have mainly aerial figures of boats.
The dataset used to test the pre-trained neural network was provided by IDEMA and consisted of an image dataset with 4000x3000 pixels that contains 214 boats.

\section{RESULTS}

Three propagation models were used to simulate the communication channel between the devices from the multi-UAV system: Free space (Eltahir, 2007), OkomuraHata (Medeisis and Kajackas, 2000) and a probabilistic model (Martins, 2006). The goal is to verify the XBee S3 module behavior in different scenarios, investigate the aircraft availability during the flight in the monitoring process and set the coverage area of each aircraft.

The communication system modeling used the following parameters: a $250 \mathrm{~mW}$ power transmission, the aircraft flight altitude of $90 \mathrm{~m}$, the base station has a 1.5 height relative to the ground and both $\mathrm{BS}$ and UAV has a 1.5 $\mathrm{dBi}$ antenna gain. The shadowing effects were disregarded, since the aircrafts work with a direct view between them.

\subsection{Models Performance}

The results for the proposed models are shown in Figure 5. The Okomura-Hata presented the worst performance, which is plausible, since this model calculates the path loss considering that there are buildings in the propagation space. However, the model shows that, at distances up to $1 \mathrm{~km}$, it is possible for range communication.

The Free space model was also considered inadequate because it disregarded the reflection effects from relief and demand that the antennas are fully aligned, which is a limiting factor for communication between UAVs considering that such a requirement would limit the movement of an aircraft during a mission.

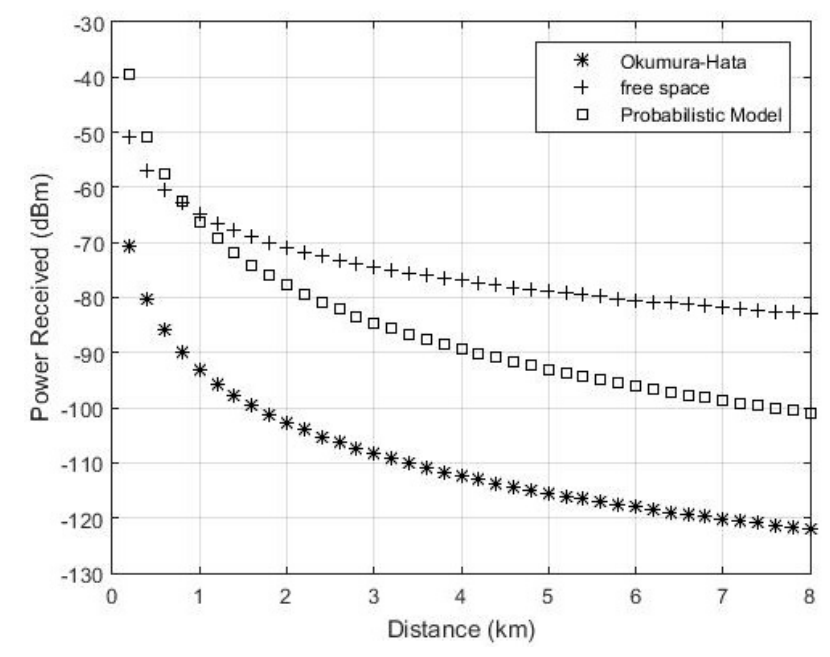

Figure 5. Path loss simulated for propagation models.

Finally, the probabilistic model presented the best results, since it was based in measures from Natal-RN, a city that has similar characteristics with the region where the UAV flight will be executed. The XBee S3 works with up to -100 $\mathrm{dBm}$ power reception. Therefore, considering the results for the probabilistic model according to Figure 5, it is possible to maintain a distance of up to $7 \mathrm{~km}$ between UAVs or UAV and base station. 


\subsection{In-flight Multi-UAV System layout}

The probabilistic model was chosen to model the APARC environment. In order to guarantee a satisfatory communication between the UAVs and between an UAV and the $\mathrm{BS}$, we set the maximum distance between these devices at $6 \mathrm{~km}$, since the simulation allowed up to $7 \mathrm{~km}$. Considering the situation where the aircrafts fly in a side by side formation, the distance between the base station and the farthest UAV will be approximately up to $6.3 \mathrm{~km}$, as illustrated em Figure 6. This value is related to the FOV from de camera and the flight altitude from UAVs.

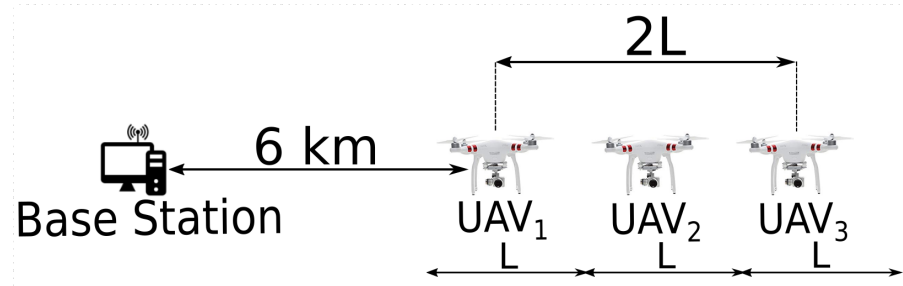

Figure 6. Distance from base station to multi-UAV system.

Figure 7 shows the decreasing signal intensity $(\mathrm{dBm})$ of the XBee communication interface from the UAVs and the base station (located at left) in a top-view perspective. The omnidirectional antennas allow the UAV to transmit signals with the same intensity and without any dependency of the flight direction. The lighter regions represent areas with higher signal intensities.

In this scenario, the UAVs can be up to $6 \mathrm{~km}$ far from each other, enabling a higher area coverage of up to $18 \mathrm{~km}$ from the base station. In this configuration, it can be observed that the BS needs to use $\mathrm{UAV}_{1}$ as a router to communicate with others UAVs, situation ilustrated in scenario (A) and (B). Similarly, scenarios (C) and (D) show that $\mathrm{UAV}_{2}$ and $\mathrm{UAV}_{3}$ can not send messages to BS without $\mathrm{UAV}_{1}$.

\subsection{Image Processing Subsystem}

To evaluate our algorithm, we set the pre-trained network to detect only boats in the image with a precision level that varies from $80 \%$ to $20 \%$ and divided the original images, that have $4000 \times 3000$ pixels, in resolutions that vary from $125 \times 125$ to $500 \times 500$ pixels. This strategy made it possible to evaluate such model with significant results. Table 2 shows the hit rate percentage of detection and Table 3 shows the amount of false positive detected in the processing.

Table 2. Hit Rate of Boats

\begin{tabular}{|c|c|c|c|c|}
\hline & \multicolumn{4}{|c|}{ Precision (\%) } \\
\hline Resolution & 80 & 60 & 40 & 20 \\
\hline $500 \times 500$ & 4.67 & 11.21 & 22.43 & 41.59 \\
\hline $300 \times 300$ & 20.09 & 37.38 & 47.19 & 62.15 \\
\hline $250 \times 250$ & 19.62 & 28.50 & 35.51 & 49.06 \\
\hline $125 \times 125$ & 10.75 & 19.62 & 28.97 & 40.18 \\
\hline
\end{tabular}

Analyzing Tables 2 and 3, it can be seen that the two best outputs achieved uses 300x300 pixels. The difference is in the precision rate: a $20 \%$ precision rate results in $62.15 \%$ of boats detection but shows 10 false positives, while a $40 \%$ precision rate results in a $47.19 \%$ of boats detection and only 1 false positive.
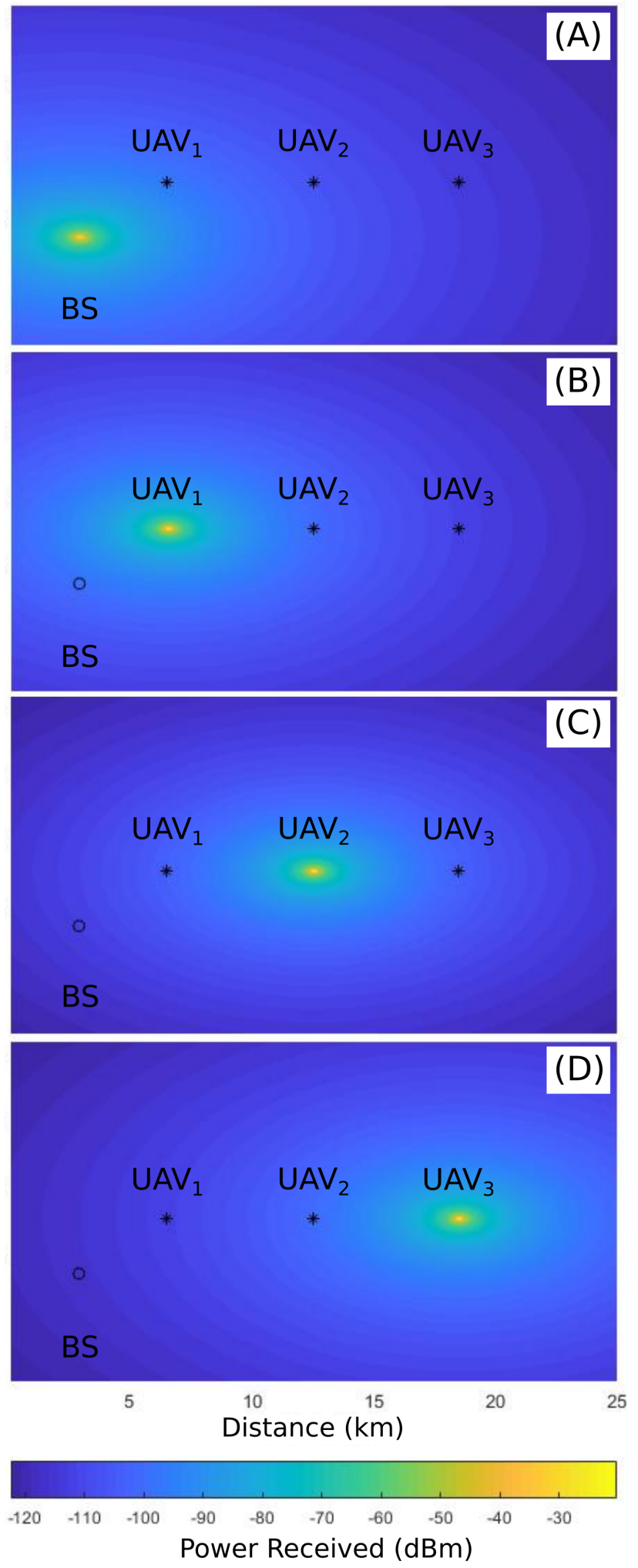

Figure 7. Multi-UAV system disposition during the flight and the power received distribution produced by each $\mathrm{UAV}$ and base station(BS).

The Figure 8 shows one of the best detections made in our dataset images. The neural network was able to detect 12 of the 13 boats available. The red rectangles indicates the place of the boats detected. 
Table 3. False Positives

\begin{tabular}{|c|c|c|c|c|}
\hline & \multicolumn{4}{|c|}{ Precision (\%) } \\
\hline Resolution & 80 & 60 & 40 & 20 \\
\hline $500 \times 500$ & 0 & 0 & 0 & 0 \\
\hline $300 \times 300$ & 0 & 0 & 1 & 10 \\
\hline $250 \times 250$ & 0 & 0 & 2 & 17 \\
\hline $125 \times 125$ & 0 & 1 & 3 & 28 \\
\hline
\end{tabular}

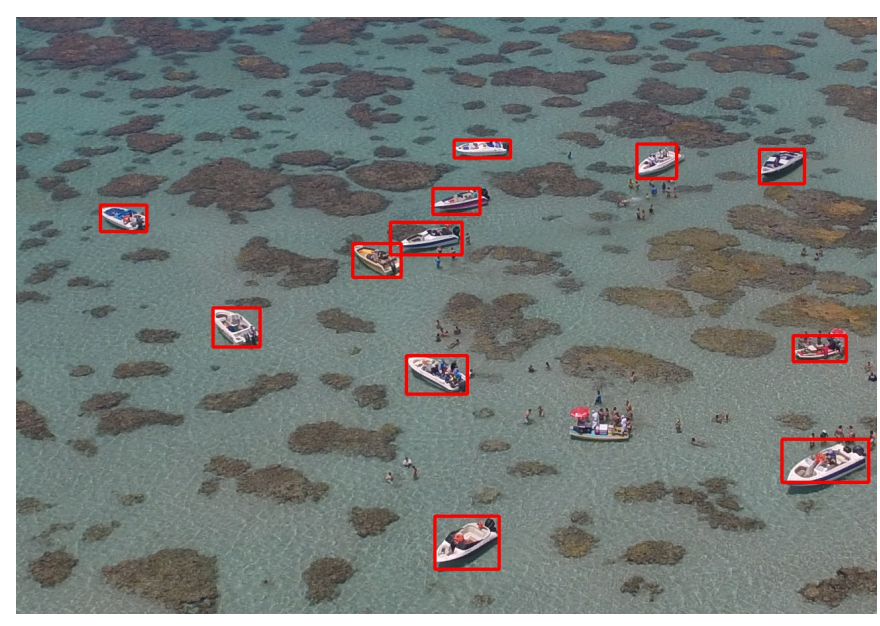

Figure 8. Boat detection

\section{CONCLUSIONS}

In this paper we presented a multi-UAV monitoring system to detect human activities in environmental protection areas. Our main contribution is the development of a new communication architecture focused in UAV for monitoring tasks and the incorporation of an image processing algorithm in the UAVs that uses the SSD neural network to detect boats. Furthermore, a satisfactory strategy to capture images from the UAVs was defined, different propagation models in the radio link were simulated to define the maximum distance between the system components and the neural network was tested using a real dataset provided by IDEMA.

The simulations prove that the XBee S3 module allows for up to $6 \mathrm{~km}$ between communication nodes without compromising the performance of the system. The creation of intersection areas between the images captured by the UAVs introduce more reliability to the system, since these areas will be checked twice. The SSD neural network presented satisfactory results, mainly when the image resolution was $300 \times 300$ pixels with $20 \%$ of precision.

In the future works, we plan to train our own neural network with only aerial views using the SSD framework, so that we may improve the hit rate detection. Thereafter, we will perform field experiments to validade the approach presented in this paper.

\section{ACKNOWLEDGEMENTS}

This study was financed in part by the Coordenação de Aperfeiçoamento de Pessoal de Nível Superior - Brasil (CAPES) - Finance Code 001.

The images used in this study to evaluate the boat detection were provided by Daniel Maciel - IDEMA.

\section{REFERENCES}

Balampanis, F., Maza, I., and Ollero, A. (2017). Coastal areas division and coverage with multiple uavs for remote sensing. Sensors, $17(4), 808$.

BRASIL, L.E.I. (2000). N 9.985, de 18 de julho de 2000. Regulamenta o art. 225, §1o, incisos I, II, III e VII da Constituição Federal, institui o Sistema Nacional de Unidades de Conservação da Natureza e dá outras providências., volume 19. Congresso Nacional.

Chen, Y., Zhang, H., and Xu, M. (2014). The coverage problem in uav network: A survey. In Computing, Communication and Networking Technologies (ICCCNT), 2014 International Conference on, 1-5. IEEE.

Dunbabin, M. and Marques, L. (2012). Robots for environmental monitoring: Significant advancements and applications. IEEE Robotics \& Automation Magazine, 19(1), 24-39.

Eltahir, I.K. (2007). The impact of different radio propagation models for mobile ad hoc networks (manet) in urban area environment. In The 2nd International Conference on Wireless Broadband and Ultra Wideband Communications (AusWireless 2007), 30-30. IEEE.

Everingham, M., Van Gool, L., Williams, C.K., Winn, J., and Zisserman, A. (2010). The pascal visual object classes (voc) challenge. International journal of computer vision, 88(2), 303-338.

IDEMA (2019). h: / www.idema.rn.gov.br. 2019.

Liu, W., Anguelov, D., Erhan, D., Szegedy, C., Reed, S., Fu, C.Y., and Berg, A.C. (2016). Ssd: Single shot multibox detector. In European conference on computer vision, 21-37. Springer.

Martins, R.d.A. (2006). Modelagem e medições de ondas de rádio para predição de perda de propagação em ambientes urbanos. UFRN.

Medeisis, A. and Kajackas, A. (2000). On the use of the universal okumura-hata propagation prediction model in rural areas. In Vehicular Technology Conference Proceedings, 2000. VTC 2000-Spring Tokyo. 2000 IEEE 51st, volume 3, 1815-1818. IEEE.

Papatheodorou, S., Tzes, A., and Stergiopoulos, Y. (2017). Collaborative visual area coverage. Robotics and Autonomous Systems, 92, 126-138.

Roldán, J.J., Joossen, G., Sanz, D., del Cerro, J., and Barrientos, A. (2015). Mini-uav based sensory system for measuring environmental variables in greenhouses. Sensors, 15(2), 3334-3350.

Santos, C., Costa, D., Junior, V., Silva, B., Leite, D., Junor, C., Liberator, B., Nogueira, M., Senna, M., Santiago, G., et al. (2015). Scanning rocket impact area with an uav: First results. In 22nd ESA Symposium on European Rocket and Balloon Programmes and Related Research, volume 730, 221.

Silva, B.R.F., Nogueira, M.B., Santiago, G.S., Dantas, J.B.D., Albuquerque, G.L.A., Alsina, P.J., and Medeiros, A.A.D. (2015). Sistema de visão computacional para detecção de embarcações por veículo aéreo não tripulado. In XII Simpósio Brasileiro de Automação Inteligente (SBAI), volume 22, 1730-1735. 